\title{
La capacitación de gestores para el desarrollo en el medio rural en México
}

A formação de gestores para o desenvolvimento rural no México

The training of managers for development in rural Mexico

La formation des cadres pour le développement dans les régions rurales du Mexique

\author{
José Angel Vera Noriega* \\ Claudia Karina Rodríguez Carvajal*
}

Recebido em 2/2/2010; revisado e aprovado em 10/6/2010; aceito em 11/12/2010

\begin{abstract}
Resumen: El objetivo de este ensayo es analizar la propuesta que Financiera Rural bajo responsabilidad académica de Colegio de Posgraduados para la formación de Prestadores de Servicios como Gestores de Desarrollo bajo una metodología constructivista trabajo-aprendizaje. Discutir por otro lado, algunos aspectos de planeación y evaluación relacionados con el plan de negocios y la definición de proyecto estratégico y finalmente, proponer algunos puntos para la preparación integral de los gestores de desarrollo que alineado al Plan Nacional de Desarrollo que contribuya con la colocación de crédito, apoyo y servicios para el campesino rural.

Palabras clave: Gestores de desarrollo. Trabajo y aprendizaje. Proyecto estratégico.

Resumo: O objetivo deste trabalho é analisar a proposta da Escola de Pós-graduação em ciências agrárias para a formação de prestadores de serviços, tais como gerentes de desenvolvimento de trabalho em uma metodologia construtivista de aprendizagem. Discutir, por outro lado, alguns aspectos do planejamento e avaliação relacionada com o plano de negócios e definição de projetos estratégicos e, finalmente, propor alguns pontos para o desenvolvimento integral dos gestores de desenvolvimento alinhado com o Plano de Desenvolvimento Nacional para ajudar com a colocação crédito, apoio e serviços para o produtor rural.

Palavras-chave: Gestores de desenvolvimento. Trabalho e aprendizagem. Projeto estratégico.

Abstract: The objective of this essay to analyze the proposal of Rural Financial under responsibility of the PostGraduates College for the formation of Service Providers as Development Managers under a working-learning constructive methodology. On the other hand, to discuss some aspects of planning and evaluation related with the business plan and the definition of strategic plan; finally, to propose some aspects for the integral preparation of development managers that contributes with the Mexican Development Plan, providing funding and support for rural farmers.

Key words: Development managers. Working and learning. Strategic project.

Résumé: Le but de cet article est d'analyser le projet de Financiera Rural, sous la responsabilité académique de Colegio de Posgraduados pour la formation des prestataires de services comme un gérant de développement sur une méthodologie constructiviste de travail et d'apprentissage. Discuter d'autre part, certains aspects de la planification et l'évaluation relatives au plan d'affaires et de la définition du projet stratégique et, enfin, propose quelques points pour le développement intégral de gérance en développement alignée sur le Plan National de Desarrollo pour aider au placement de crédit, d'assistance et de services pour les agriculteurs en milieu rural.

Mots-clés: Gérants de développement. Travail et d'apprentissage. Projet stratégique.
\end{abstract}

\section{Introducción}

La Financiera Rural tiene por objetivo, consolidar un sistema de financiamiento y canalización de recursos financieros, asistencia técnica, capacitación y asesoría en el sector rural. Además, propiciar condiciones para la recuperación del nivel de vida en el medio rural mediante la oferta de sus productos, en beneficio de los productores rurales, sobre todo a través de entidades intermediarias que aseguren el impacto en ellos (FINANCIERA RURAL, 2008a).
La Financiera Rural (FR) con el objeto de hacer más eficiente su operación, pone en manos de actores particulares el proceso a través del cual se desarrolla y solicita apoyos para las diferentes acciones que Financiera esta promoviendo con los productores. La figura del Prestador de Servicios Profesionales (PSP) se asocia a través de despachos asociados que se encargaran del diseño, desarrollo y evaluación de los planes de negocios. El Prestador de Servicios Profesionales (PSP) en las regiones rurales de México funciona como un mediador entre los intereses y planes estratégicos de la empresa y el plan de negocios a las en-

\footnotetext{
* Professores Doutores do Centro de Investigación en Alimentación y Desarrollo, A.C., Carretera a la Victoria km. 0.6,
} Hermosillo, Sonora, CP 83000, AP 1735. Teléfono: (662) 2892400 ext.317. E-mail: avera@ciad.mx, psicología@ciad.mx. 
tidades privadas o públicas en la búsqueda de financiamiento. El Gestor de Desarrollo Rural llamado PSP además orienta con métodos participativos y analíticos el posicionamiento de la organización productiva frente a la cadena productiva, de valor y la integración económica, regional y nacional. Por lo anterior, resulta imprescindible la actualización, mejoramiento y capacitación de esta figura central en el andamiaje estructural y funcional del financiamiento al campo, en una época en la cual las reglas del mercado, comercialización y financiación han modificado radicalmente, introduciendo no solo las nuevas tecnologías de la información y la comunicación, sino avances agroindustriales derivados de las innovaciones en procesos, productos y sistemas, además de aquellos asociados a los cambios en la conceptualización y entendimiento de los territorios rurales.

\section{El nuevo paradigma de desarrollo rural}

Actualmente se concibe el Desarrollo Rural Sostenible (DRS) como un proceso de transformación de las sociedades rurales y sus unidades territoriales, centrado en las personas, participativo, con políticas específicas dirigidas a la superación de los desequilibrios sociales, económicos, institucionales, ecológicos y de género, que busca ampliar las oportunidades de desarrollo humano (TORRES, 2006).

Algunas estrategias básicas para un desarrollo rural sostenible son: reducción de la pobreza rural, concebir y aplicar modelos eficientes de planificación territorial, descentralizados y democráticos, que consideren los aspectos del desarrollo productivo, social, cultural y ecológico de las regiones o espacios rurales y sus múltiples interrelaciones con centros urbanos y comunidad nacional (DOUROJEANNI, 2000).

El DRS, está determinado por una combinación que optimice el capital financiero, humano y social. Además de los recursos económicos y los procesos de inversión y gastos de las organizaciones productivas, el sector rural cuenta con capital social (KLIKSBERG, 1999), que expresa sólidos procesos históricos de construcción de comunidades y naciones. Este capital social se relaciona con la confianza, solidaridad y participación de las poblaciones que se expresa en sus ritos, mitos y formas de organización tradicional. Ello significa una vasta experiencia en la construcción de redes y sistemas de apoyo solidario que es importante rescatar para emprender la tarea de promover un nuevo modelo de mediación a través del PSP para colocar al alcance los productos de Financiera Rural bajo un paradigma del desarrollo sostenible (MORA, 2002).

El nuevo Desarrollo Rural Sostenible requiere de estrategias capaces de modificar la estructura de la demanda, incorporando al $85 \%$ de los productores rurales que se encuentran en la producción primaria en los eslabones de la cadena productiva de abasto, acopio, almacén, transporte, comercialización y financiamiento. Esto permitirá generar procesos de integración económica y resolver la atomización de las unidades productivas.

Sin embargo, la naturaleza heterogénea de la sociedad rural, de sus sistemas productivos, entorno ecológico y de su cultura, plantean la necesidad de formular políticas diferenciadas que reconozcan las condiciones particulares y las potencialidades de cada grupo y den respuestas a las demandas de los territorios sobre los procesos y procedimientos de integración y desarrollo.

En el plano agro empresarial, deberán tenerse presente los intereses, potencialidades y medidas orientadas a los grandes, pequeños y medianos empresarios agropecuarios y forestales, la empresa familiar campesina con énfasis en la microempresa y agroindustria, la industria y los servicios que se ubican en los espacios rurales. En el plano socioeconómico, es necesario ejecutar acciones explícitas hacia y en favor de las mujeres, los jóvenes, las etnias y otros grupos vulnerables y excluidos, favoreciendo el desarrollo de sus potencialidades y considerando aspectos de equidad, perspectiva de género, respeto a la diversidad cultural, diferencias de edades, desigualdad de condiciones socioeconómicas, entre otros (GONZÁLEZ, 2009).

Sin desmerecer la importancia de todos los grupos, poblaciones y expresiones productivas existentes en el medio rural y la pertinencia de políticas diferenciadas para cada uno de ellos, destacamos los siguientes: juventud rural, mujeres rurales y empresa familiar campesina con énfasis en microempresa y agroindustria. 
Se trata de generar acciones que faciliten el acceso a la tierra, agua, servicios y bienes públicos, financiamiento rural, asistencia técnica y tecnología. El propósito es aumentar las capacidades de la sociedad rural para impulsar un proceso de crecimiento integral, incluyendo acciones que posibiliten el empoderamiento de la sociedad civil rural y la adecuación del marco institucional que le apoye y promueva. La consecución de un desarrollo sostenible exige la participación real y activa de todos los grupos y de sus organizaciones, incluidas las mujeres, los jóvenes, los pueblos indígenas y sus comunidades, las comunidades locales, las organizaciones no gubernamentales, los agricultores, las autoridades locales, los trabajadores y sus organizaciones, el comercio y la industria y la comunidad científica y tecnológica (SCHJTMAN; BERDEGUÉ, 2004).

Para lograr lo anterior se requiere una iniciativa de mega planeación estratégica (KAUFMAN, 2004) que permita a los territorios rurales diseñar, incubar y fortalecer un corporativo de empresas que integren distintas fases de la cadena productiva permitiendo procesos de la integración económica, a través de generar micro finanzas, entidades dispersoras de crédito e intermediarios financieros rurales.

\section{La formación del gestor del desarrollo}

Colocar a nuestros territorios rurales en el nuevo paradigma del Desarrollo Rural Sostenible requiere aumentar las capacidades organizativas de los colectivos, organizaciones, grupos y a su vez promover conducta participativa orientada a la autogestión y autodeterminación a través de un proceso reflexivo y crítico sobre la gobernabilidad y procesos institucionales, en donde los intereses personales, egocentrismo e intolerancia son repertorios que obstaculizan el avance de las iniciativas actuales hacia el desarrollo integral. Particularmente, enfocado a la estructura y funcionalidad de los procesos organizacionales sus perfiles, estilos y diversidad con el objeto de mejorar las aptitudes productivas y transformar los indicadores de competitividad de las comunidades. En este contexto socioeconómico, la figura del gestor de desarrollo (PSP) aparece como un actor fundamental que con la preparación y actualización bajo un modelo educativo andragógico estructurado bajo los principios de las evidencias actuales de la neuropsicología del aprendizaje, antropología social y sociología rural pueda jugar el papel de mediador entre las posibilidades del desarrollo del territorio y las de la organización de productores.

El profesional deberá ser una persona con capacidad de intervención y habilidades de gobernar o controlar los procesos de gestión y relación entre los sistemas que se relacionan, asumiendo la postura de un animador económico - social. Estas personas deberán formarse como emprendedores (estrategias para definir proyectos, delimitando riesgos) y operadores territoriales que conozcan y operen eficazmente sobre el territorio, desde los diferentes sistemas y subsistemas y manejar didácticas activas como el aprendizaje por competencias, colaborativo y significativo. La enseñanza deberá tener lugar en el contexto de las organizaciones y será necesario capacitar a la persona especialista en desarrollo local a elaborar ajustes o transferencias de conocimiento abstracto a concreto a través de los diferentes escenarios sociales y económicos que requiere la planeación de futuro de la integración económica regional (AGUILERA; JIMÉNEZ, 2007).

Además, se requiere establecer competencias de gestión y sectoriales. Las competencias de gestión para desarrollar habilidades en el estudiante para su relación con agentes $\mathrm{y}$ actores institucionales productivos, de investigación; y sectoriales, vinculados a los procesos y prácticas medulares en las especializaciones productivas territoriales.

Considerando por un lado, la necesidad de apoyo de los productores rurales con asistencia técnica integral para mejorar la utilización de los créditos y por otro lado, transformar los procesos organizacionales para constituir, operar y fortalecer los intermediarios financieros rurales, la Financiera Rural diseña y coloca en operación la Maestría en Prestación de Servicios Profesionales (MPSP) en convenio con el Colegio de Posgraduados (COLPOS) y con convenios asociativos en toda la nación para preparar a los Gestores de Desarrollo (PSP) en un nuevo modelo de gestión y competencias para clientes y usuarios de la Financiera Rural. 


\section{Maestría en Prestación de Servicios Profesionales (MPSP)}

El objetivo de la MPSP es formar capacitadores, asesores y consultores rurales en activo, en los fundamentos teóricos de los procesos de aprendizaje, en los fundamentos metodológicos que derivan de ellos para la capacitación rural, así como en las capacidades teóricas y prácticas para diseñar realizar, retroalimentar y evaluar procesos de aprendizaje que generen las competencias laborales y soluciones tecnológicas requeridas para el diseño, incubación y fortalecimiento de empresas e intermediarios financieros rurales (FINANCIERA RURAL, 2004).

El programa busca ofrecer a los prestadores de servicios una formación actualizada y de carácter integral, que conlleva un enfoque interdisciplinario de la capacitación y del aprendizaje, en el cual concurren diversas ramas del conocimiento científico por sus aportes al objeto de estudio.

La maestría en PSP entiende el desarrollo rural de la siguiente manera:

El desarrollo como estrategia debe buscar necesariamente ser sustento de la sociedad que lo promueve, al propio tiempo todo enfoque de desarrollo que no pueda ser sostenido por la sociedad que lo promueve resulta excluyente y por ende puede preverse su fracaso a mediano o largo plazo. De esta manera, se trata de promover aquel tipo de desarrollo que pueda ser conducido y realizado por la sociedad a la que sirve, no solo por razones de objetivo social sino porque además el desarrollo exógeno resulta un contrasentido. (FINANCIERA RURAL, 2010, p. 29).

Lo anterior considerando los aspectos de corto y largo plazo y no sacrificando el medio y los recursos ambientales por el beneficio económico.

Finalmente, el enfoque del desarrollo rural que busca imprimir el programa de formación considera a los productores como personas capaces de llevar a cabo procesos de autogestión del desarrollo determinando los proyectos y procesos productivos que habrán de impulsar, los cambios tecnológicos y los objetivos mismos de los proyectos (FINANCIERA RURAL, 2010).

La maestría está compuesta de 5 módulos que se cursan vinculados a un proyecto estratégico de financiera rural para cada alumno. Un modulo contiene 4 cursos, cada uno de ellos genera insumos para lograr el objetivo que persigue el proyecto estratégico encada una de sus unidades de negocios. Al terminar el cuarto modulo el alumno que a su vez es un gestor del desarrollo ha incorporado los principios del modelo de prestación de servicios de financiera, el cual implica: autonomía y autogestión de las organizaciones productivas, formación de alianzas estratégicas a través de redes que permiten apropiarse de la cadena de valor e integrarse a la economía regional, unidades de negocios que se logran a partir del capital social y sustentabilidad del sujeto de crédito.

\section{El Modelo Pedagógico de la Maestría en Prestación de Servicios Profesionales}

El modelo pedagógico trabajo-aprendizaje (M/T-A) de la Maestría en Prestación de Servicios Profesionales de Financiera Rural que constituye la base técnica que da origen a un andamiaje curricular dividido en 5 módulos alineados a normas de competencia laboral, está planteado desde la perspectiva de responder al plan de vida de una empresa desde un modelo de gestión de la calidad con un análisis micro y macro que corresponde a los niveles de interiorización de los procesos productivos en las empresas. Los recursos humanos que se están formando en este proceso de financiera rural intentan establecer conocimientos, habilidades y competencias para el comportamiento individual y colectivo en el contexto de las empresas conllevando cambios en las estructuras organizacionales e institucionales que les permitan hacerse mucho más competentes en su ramo y estar en condiciones de enfrentar los retos de las nuevas tecnologías en la sociedad del conocimiento (GARZA, 2006).

$\mathrm{El} \mathrm{M} / \mathrm{T}-\mathrm{A}$ se caracteriza por ser un sistema de formación en el que el alumno avanza a través de algunos estadios de diferente complejidad e inclusivos desde el conocimiento hacia la habilidad entendida como un proceso integrador y sintético para ejecutar un procedimiento y la capacidad entendida como diferentes habilidades relacionadas con la toma de decisiones, la solución de problemas hasta el pensamiento estratégico.

El M/T-A es un paradigma de gran 
complejidad, pues requiere de una serie de axiomas o principios como la autogestión de aprendizaje, el aprendizaje colaborativo y el aprendizaje significativo. Una de las condiciones que son fundamentales para modelo de trabajo-aprendizaje es que el aprendiz sea acompañado de un experto el cual, deberá ser competente en aquellas habilidades que pretende enseñar. Una condición adicional es que el alumno deberá estar involucrado en el contexto o escenario en el que tiene lugar el aprendizaje y corresponde con el escenario en el que se presenta el problema.

El profesional necesita reconocer, asimilar y analizar las características didácticas de cada uno de las formas de producción rural y su relación con las vocaciones territoriales. Esto es, las relaciones entre los diferentes eventos físicos, biológicos y sociales en la realidad que caracteriza la dinámica procesual de un evento constituido socialmente. Sembrar maíz, ordeñar vacas, producir miel, sacrificar ovinos, transformar la madera, comercializar transportes y muchos más no corresponden de manera unitaria a una disciplina y requieren para su estudio de un conjunto de disciplinas que de manera integral sean capaces de explicar la forma histórico-social de la construcción de los tipos de producción rural. Sin embargo, la explicación de este fenómeno tiene un pensamiento ingenuo o ritual muchas veces bastante acabado y apegado a las variables y factores que le dan existencia al evento observado (PINA; REYES; QUESNEL, 1999).

El agricultor, el ganadero, el comerciante y cada uno de los empresarios de organizaciones civiles, privadas, productivas, sociales o de servicios que tienen amplia experiencia en el análisis y manejo de cada una de las variables y factores asociados al proceso productivo y comercial, tienen la capacidad de reconocer la didáctica del fenómeno. El modelo pedagógico parte de que la didáctica del fenómeno ha sido construida socialmente, que debe ser analizada partiendo del análisis cotidiano de la experiencia del productor e incorporar datos y métodos de la ciencia a través de la interacción continua bajo un modelo de gestión de calidad (FINANCIERA RURAL, 2006, p. 41).

Supone este modelo que el desarrollo de la ciencia, la innovación y la tecnología pueden acarrear beneficios sustantivos a la competitividad empresarial siempre y cuando la competencia se entienda como un diferencial entre el estado real y el ideal en la perspectiva planificada de ontogénesis de calidad y competitividad que evalúe una relación de los riesgos que la empresa asumiría si se involucrara en una ruptura epistemológica. Esto es, el entendimiento de la didáctica de un fenómeno es y será siempre una ventaja comparativa para los productores, asumiendo un desarrollo tecnológico o sistema de innovación que permita mejorar la competitividad del proceso, producto o sistema productivo. Sin embargo, cambiar la estructura organizacional, integrando consorcios, clústeres, corredores o en su caso tecnificar los procesos de producción primaria o innovar sobre el producto requiere que el pensamiento humano se dirija hacia nuevas alternativas, hacia nuevas relaciones criticas que establezcan condiciones para el manejo y producción de un bien social que sirva para mejorar la calidad de vida para el contexto local o regional.

El modelo curricular de Financiera Rural parte de una serie de acciones sobre los avances actuales de la neuropsicología del aprendizaje en los que se supone: a) que nuestro contacto con los eventos rutinarios y cotidianos requiere de la conformación de redes; b) el sistema nervioso central está diseñado de tal manera que los organismos pueden ser impactados por recompensas simples en períodos de tiempo cortos más que por recompensas complejas en tiempos largos; c) El sistema nervioso central está organizado y se desarrolla de tal manera que las redes neuronales se vuelven más complejas al estar desarrollando habilidades de pensamiento estratégico. Una derivación de este presupuesto se refiere a la necesidad de evitar lo desechable, lo efímero, lo intrascendente y fijarse metas vinculadas con la solución de problemas y la toma de decisiones en situaciones complejas que impliquen un alto grado de conocimiento y un número de variables y factores; d) Los circuitos cerebrales que hacen posible el pensamiento complejo potencialmente pueden ser desarrollados por cualquier ser humano. No existen diferencias por raza edad sexo o ninguna otra variable que haga imposible que el equipo biológico pueda desarrollarse en toda su plenitud y 
potencialidad haciendo posible la innovación y desarrollo; e) Las diferencias en las potencialidades se desarrollan a partir del contexto de estimulación en el cual nos desarrollamos y construimos socialmente; f) Las diferencias en el desarrollo psicosocial están dadas por el contexto y se van generando de manera inclusiva desde la infancia hasta la adultez, entre menos posibilidades existan para el enriquecimiento ambiental y el pensamiento productivo, el consumo cultural, calidad de vida y estilos, las redes neurales serán un reflejo del contexto social de cada persona (VERA; PEÑA, 2005).

El aprendizaje se construye socialmente y tiene lugar a través del organismo individual como un proceso inclusivo, evolutivo y de incremento de complejidad, esto implica reconocer que una organización social priva$\mathrm{da}$, productiva de servicios tiene un capital humano asociado a sus potencialidades, no sólo en términos de desarrollo individual de cada uno de ellos, de sus estudios, de sus edades, de sus experiencias tempranas, de los contactos escolarizados, semi-escolarizados e informales sino que además, como colectivo se genera una unidad de pensamiento grupal, un plan de vida organizacional e institucional y se aprende momento a momento y año con año a vivir en armonía, en convivencia y en confianza. Así pues, el capital social entendido como confianza, solidaridad y participación tiene su origen y basa sus posibilidades en aquellas del capital humano, el cual está implicado en los aspectos individuales ontogenéticos de cada uno de los componentes de la organización (KLIKSBERG, 2000).

En resumen, el modelo pedagógico trabajo-aprendizaje vinculado a otro modelo conceptual sobre los procesos de percepción, evaluación, planeación, solución de problemas y desarrollo psicológico y social de los agentes y actores de los procesos de integración económica que se encuentra bajo los principios, leyes y supuestos de las teorías mas actuales del constructivismo de la psicología y sociología, los modelos estructurales de la antropología social, la semiótica y los hallazgos actuales de la neuropsicología del aprendizaje.

\section{Definición del proyecto estratégico}

El modelo de Financiera Rural para la Formación de Prestadores de Servicios Profesionales (M/T-A) incluye todos estos principios y requiere asumir los procesos de desarrollo individual de la organización social y tener presente que el aprendizaje es una construcción social relacionada con el individuo así como la didáctica es una construcción social relacionada con el fenómeno $u$ objeto de estudio y que en ambos casos se requiere promover el entendimiento y la profundización del conocimiento sobre el evento para hacer posible los ajustes y cambios a la empresa para la satisfacción del cliente.

Desde la perspectiva de Financiera Rural el concepto de proyecto estratégico: a) es una iniciativa planificada de una o varias organizaciones de productores para diseñar, incubar y fortalecer un corporativo de empresas; b) las empresas dibujan una cadena de producción, abasto, servicios; c) el proyecto permite el establecimientos de procesos de integración económica (FINANCIERA RURAL, 2008b), d) es un planteamiento similar al modelo de gestión de calidad de Stuffebeam e Shinkfield (1993), el cual está orientado al cliente y requiere un análisis de insumo, proceso y producto y su relación con las fortalezas, debilidades, oportunidades y amenazas. Este modelo se orienta particularmente hacia el cliente y el objetivo fundamental del modelo es mejorar la competitividad. Entendiendo competitividad como un diferencial entre lo que es y lo que será, relacionado con el cliente, estableciendo así la condición para la mejora continua en el futuro inmediato o a corto plazo. En este modelo el plan de vida empresarial se sustenta y se concentra con alcanzar metas a corto plazo que hagan más rentable los procesos productivos (TORRES, 2006).

La propuesta de planeación estratégica debería transformarse hacia una mega planeación que requiere orientar inicialmente los principios de la planeación hacia fines sociales, en donde las iniciativas de los corredores industriales, de los clúster y de las cadenas productivas no sólo se enfocan a la mejora del mercado, mercancía o del mercader, sino buscan incluir en su misión y visión intereses sociales que hagan de su actividad productiva 
una actividad más humana, colectiva, equitativa e igualitaria para que el proceso sea integral (KAUFMAN, 2004).

Esta consideración es relevante porque toda vez que sea incluida en el plan formativo del gestor del desarrollo deberán incorporarse nuevos aspectos de la planeación estratégica micro y macro porque ambas estarán orientadas al bienestar y la felicidad común. Es fundamental entonces atender a los presupuestos de una mega planeación, la cual pone su interés no en un estatus de felicidad o bienestar, sino en lo que la empresa puede ofrecer a través de sus productos para el bienestar común dejando atrás la idea de que la mejora continua está referida a cambios operativos o instrumentales. Un modelo basado en la mega planeación supone que la mejora continua es una gestión del día a día y que tiene como objetivo el cambio de los indicadores y propósitos hacia el interés como organización productiva y de servicios de atender la mejora de los indicadores de desarrollo local y territorial.

Desde esta nueva perspectiva el plan de negocios requerirá también algunas adiciones a sus presupuestos como por ejemplo, asumir que la didáctica como construcción social de un fenómeno es imperativa y está presente a través de las diferentes generaciones y consideran a un producto y/o servicio como importante para la sociedad cuyo objetivo es no sólo resolver el problema de una persona, sino remontarse a los indicadores más sociales e intentar la incorporación de metas que redunden en la mejora continua de la calidad de vida de los ciudadanos.

La diferencia entre la planeación estratégica y la mega planeación implica estar generando impacto social de futuro y conjuntar los intereses de las organizaciones a través de la planeación estratégica y llevar a cabo arreglos anticipatorios para responder a las necesidades y mejora de la sociedad. Además debe entenderse que la ganancia económica es menos importante que el capital social, la convivencia, la armonía que beneficien a los grupos mayoritarios estableciendo las condiciones para un progreso conjunto en el territorio o localidad y generando un sistema de educación ciudadana para la democracia.

El diseño de proyectos estratégicos dentro de Financiera Rural requiere evaluaciones estratégicas en el territorio y de la capacidad de capital social para las organizaciones y el desarrollo productivo. Los proyectos estratégicos que son asumidos por estudiantes de la Financiera Rural implican el conocimiento sobre los procesos productivos y el desarrollo organizacional y se enfocan a colocar estrategias y técnicas innovadoras y tecnológicas para que la empresa logre mayor competitividad y riqueza (UC-HERNÁNDEZ; MATUS, 2006).

Sin embargo, esta visión de proyecto estratégico requiere agregar indicadores de fortalecimiento de las instituciones, desarrollo organizacional, y sobre todo indicadores sociales que incluyan la medición del impacto del proyecto en la mejora de la calidad de vida del territorio y la localidad. En resumen, el concepto de proyecto estratégico que plantea Financiera Rural requiere incorporar algunos aspectos de desarrollo social además de integración económica (GÓMEZ, 2007).

\section{Procedimiento para la elaboración del plan de negocio}

El procedimiento para la elaboración del plan de negocios se constituye de dos partes, una relacionada con los insumos que requiere el diseño del nuevo producto y otra relacionada con reingeniería que requiere la planta para producir el nuevo producto o servicio. La primera parte, es de suponer que en todo el momento el gestor de desarrollo orientará a la organización de empresarios hacia el rediseño del proceso y producto bajo criterios de competitividad empresarial con las herramientas tecnológicas y de innovaciones adecuadas, justas y con ventajas comparativas. El producto puede tener el mismo empaque, la misma vida de anaquel, las mismas propiedades físicas y químicas, pero lo que se modifique sean posiblemente las estrategias de acopio, de insumos o de comercialización, en fin, el modelo de negocios se propone encontrar una veta a través de la cual pueda establecer un número de posibilidades de cambio para presentar éste abanico ante la organización y sobre sus fortalezas y debilidades encontrar la solución más adecuada siempre desde el marco de un modelo de gestión de calidad (JUNKIN, 2005).

Esto hace suponer que la empresa deberá estar llamada a reinventarse sea como fuere 
en cualquiera de sus momentos o procesos, lo cual debe evaluarse pues, no todas las empresas aún cuando tengan problemáticas asociadas o competitividad, están en condiciones de desarrollar un modelo de gestión de la calidad e involucrarse en un cambio. Los principios mismos del aprendizaje individual y colectivo nos hacen ver que se requiere un basamento de experiencias para involucrarse en un proceso de cambio. Por otro lado, aún cuando muchas empresas cuenten con los insumos para involucrarse en el cambio que se anuncia, deben tener información sobre los intereses ciudadanos y sociales los cuales le dieron origen a la forma de producción y hacen probable que tal cambio organizacional o empresarial promueva la mejora en las formas de operar en el binomio empresasociedad civil.

El diseño del plan de negocios deberá iniciar con la definición del interés social de los objetivos de la empresa y no suponer que para alcanzar los indicadores sociales sólo deberá actuar a través de la calidad de sus productos sino además, pueden requerirse estrategias innovadoras como la economía solidaria, responsabilidad social, sociedad civil organizada, fondos mutualistas y el análisis de estrategias que provean la información suficiente para alcanzar otras propuestas coherentes y congruentes con la planeación.

En relación a los ajustes que se deberían incluir en la segunda parte del plan de negocios, encontramos como insumo fundamental la inclusión de una estrategia de generación y aplicación del conocimiento y no solo asuntos instrumentales y operacionales de la empresa, sino más bien desarrollo del capital intelectual que deriva de la afirmación y capacidad de sus dirigentes, sus empleados y su gerencia (ROSS et al., 2001). Los costos más relevantes no son los económicos y monetarios sino aquellos relacionados a la producción de conocimiento útil para la solución de problemas, por lo que es de primordial interés cuantificar el costo de los procesos de innovación y desarrollo que tendrán lugar a través de alianza con las universidades y centros de investigación enfocándose siempre a los mega postulados (DAVID; FORAY, 2002).

De esta forma es posible que los ajustes e inversiones instrumentales de ingeniería para la transformación del producto pasen a un segundo o tercer plano toda vez que el gestor de desarrollo deberá estar atento a aquellos conocimientos y tecnologías que son necesarias inventarse o reinventarse a través de las universidades y centros de investigación para después a través de una interfase buscar su aplicación dentro del proceso productivo. El plan de negocios no es solamente un sistema de reingeniería sino implica forzosamente la idea de la cooperación estratégica y de la generación y aplicación del conocimiento, los cuales están actualmente ausentes en los planes de negocios de nuestros prestadores de servicios.

La forma en que se diseñan actualmente los planes de negocios en la Maestría requieren transformase, pero a su vez es necesario modificar la visión del gestor de desarrollo en la maestría de prestación de servicios profesionales porque mientras se encuentre alineado un modelo de gestión estratégica conservador y convencional y un modelo de plan de negocios centrado en la riqueza y en la mejora de la empresa, el modelo pedagógico trabajo-aprendizaje no habrá cumplido con las expectativas y seguirá siendo visto desde los estudiantes como una propuesta novedosa de aprender haciendo, pero sin ninguna consecuencia innovadora sobre el desarrollo de las organizaciones y empresas.

El modelo trabajo aprendizaje requiere modernizarse para dar paso a los dos objetivos fundamentales de la nueva planeación, la organización social y la generación de conocimiento. El modelo trabajo-aprendizaje se encuentra muy cercano a reproducir las condiciones del modelo enseñanza-aprendizaje que prevaleció por mucho tiempo en los prestadores de servicios que atienden a las empresas de nuestro país y el cual se convirtió en un sistema de bajar recursos con intereses personales y egoístas.

Creemos firmemente que el modelo trabajo-aprendizaje contiene pedagógicamente la riqueza necesaria para incorporarse dentro de una mega planeación y un proyecto de plan de negocios en el que los mega postulados implicados sean los precursores del cambio y las medidas de mediación y negociación social puedan orientar a la búsqueda de conocimiento y la innovación de tecnologías dirigida a promover el bienestar social y calidad de vida en el territorio y localidad. 


\section{Propuestas para la mejora continua de la formación de gestores de desarrollo}

El basamento fundamental del éxito y fracaso de un plan estratégico empresarial con las organizaciones sociales que cumplen el perfil de apoyo de Financiera Rural requiere de un conocimiento importante sobre la estructuración y funcionalidad de las organizaciones productivas en sus diferentes modalidades y objetivos meta-colectivos más allá de las visiones administrativas o jurídicas. El desarrollo de las organizaciones situadas en el marco de referencia del usuario de Financiera Rural, requiere considerar las características sociales, económicas y de género en el que se encuentran los diferentes actores que intervienen en el proceso de comunicación. Se deben tomar en cuenta cuatro factores para incrementar la eficiencia comunicativa; a) habilidades comunicativas, b) actitudes, c) el nivel de conocimiento del tema que se trata, d) la posición que se ocupa dentro de un sistema social.

En segundo plano se requiere que los alumnos de una Maestría en Gestión de Desarrollo puedan ser capaces de desarrollar habilidades analíticas de teorías de personalidad y psicología social que permita al profesional clasificar las invariantes del comportamiento y esté en condiciones de predecir conductas que facilitan o inhiben posturas a favor del cambio, transformación o ruptura epistemológica.

En tercer plano, se hace necesario que el gestor sea capaz de establecer prácticas, rutinas y estilos de gestión que promuevan el cambio de ideas y fomenten transiciones en la forma de enfrentar el objeto y el proceso productivo. Gestionar potencialidades sociales que permitan compartir riesgos, éxitos y fracasos ante la posibilidad de transformar el terreno de la acción y redefinir el plan de vida de la empresa (ORDOÑEZ, 2004).

Finalmente se hace necesario comprender y mejorar los parámetros de medida asociados al modelo económico de Amartya Sen (SUDHIR; SEN, 2000) sobre desarrollo humano, particularmente educación, salud, seguridad social e ingreso. Esta visión innovadora de desarrollo humano integrada al sistema de mega-planeación estratégica (KAUFMAN, 2004) podría resultar en una alternativa para estimular la equidad e igualdad en el territorio, diseñar un plan estratégico local que concentre intereses multisectoriales y vocaciones en la búsqueda de un sistema de integración económica regional.

\section{Referências}

AGUILERA, Luis; JIMÉNEZ, Ivette. Red para la formación de gestores de la ciencia, la tecnología y la innovación para el desarrollo local. X Reunión de Popularización de la Ciencia y la Tecnología en America Latina y el Caribe y IV Taller "Ciencia, Comunicación y Sociedad". San José, Costa Rica, 9 al 11 de Mayo de 2007.

DAVID, Paul; FOURAY, Dominique. Fundamentos económicos de la sociedad del conocimiento. Revista Comercio Exterior, v. 52, n. 6, p. 472-490, 2002.

DOUROJEANNI, Axel. Procedimientos de gestión para el desarrollo sustentable. Santiago de Chile: CEPAL-ECLAC, ONU, 2000. Serie Manuales.

FINANCIERA RURAL. Programa Integral de Formación, Capacitación y Consultoría para productores e intermediarios Financieros Rurales. 2004. En red: <http:/ / financierarural.gob.mx/>.

Concepción general del proyecto de formación de prestadores de servicios. México: [s.n.]. 2006.

. Misión y Visión. 2008a. En red: <http://financierarural.gob.mx/fr/paginas/MisionVision.aspx>.

. Políticas de fomento y desarrollo rural. México. 2008b. En red: <http:// financierarural.gob.mx/paginas/financierarural/aspx>.

. Formación para el financiamiento del desarrollo rural. México. 2010. En red: <http:/ / www.financieraruraldide. $\mathrm{mx} /$ contenidos.php?idsubsubcontenido=4>.

GARZA, Laura. Un nuevo paradigma en la formación de recursos humanos como estrategia del desarrollo rural. En: Agricultura, Sociedad y Desarrollo, 3 (2), 155-173, 2006.

GÓMEZ, A. Idea clave: el impulso de la Responsabilidad Social de las empresas como iniciativa estratégica en la configuración de Territorios Socialmente Responsables. Propuesta presentada a la reunión del Subgrupo de Responsabilidad Social del GTN1 del 11/10/2007.

GONZÁLEZ, Hernán. La extensión y los servicios de apoyo para la agricultura en América Latina y el Caribe: Elementos para la formulación de políticas. Boletín Infotec: Sistema de información científica y tecnológica del sector agropecuario en las Américas. IICA, 2009.

JUNKIN, Ruth. Construcción de capacidades para servicios de desarrollo empresarial orientados hacia la demanda. En: CATIE, Turrialba, Costa Rica, 11-13 abril, 2005.

KAUFMAN, Roger. Planificación mega: herramientas prácticas para el éxito organizacional. [S.1.]: Universitat Jaume I, 2004.

KLIKSBERG, Bernardo. Capital Social y cultura, claves esenciales del desarrollo. Revista de la CEPAL, n. 69, p. 85-102, Diciembre 1999.

El rol del capital social y de la cultura en el proceso de desarrollo. En: KLIKSBERG Bernardo; TOMASSINNI Luciano (Comps.). Capital social y cultura: 
claves estratégicas para el desarrollo. México: Fondo de Cultura Económica, 2000. p. 19-58.

MORA, Jorge. Desarrollo rural, cambio institucional y extensión rural en Centroamérica y México. IICA, Proyecto FONTAGRO “Investigación en extensión y servicios de apoyo: hacia una agricultura sostenible en América Latina y el Caribe. San José, 2002.

ORDOÑEZ, Sergio. La nueva fase de desarrollo y el capitalismo del conocimiento: elementos teóricos. En: Revista Comercio Exterior, v. 54, n. 1, p. 4-17, 2004.

PIÑA Adelfo; REYES Rúben; QUESNEL, Erick. La metodología de trabajo-aprendizaje: capacitación para el desarrollo rural. México: Cuadernos de trabajo, INCA Rural, 1999.

ROSS, J.; ROSS, G.; DRAGONETTI, N.; EDVINSSON, L. Capital intelectual: El valor intangible de la empresa. México: Paidos, 2001.

SCHJTMAN, Alexander; BERDEGUÉ, Julio. Desarrollo territorial rural. En: Centro Latinoamericano para el Desarrollo Rural. Debates y temas rurales 1, 2004.

STUFFLEBEAM, Daniel; SHINKFIELD, Anthony. Evaluación sistémica (guía teórica y práctica). Temas de educación. Barcelona: Editorial Paidos, 1993.
SUDHIR, Anand; SEN, Amartya. Human Development and Economic Sustainability. World Development, v. 28, n. 12, p. 2029-2049, 2000.

TAMAYO, Shirley; GARCÍA, Mariela. Estrategia estatal para el fortalecimiento de entes prestadores de servicios públicos en el pequeño municipio y la zona rural. El programa cultura empresarial adelantado en Colombia. En: QUIROZ, Franz; FAYSSE, Nicolás; AMPUERO, Raúl (Comps.). Apoyo a la gestión de comités de agua potable. Bolivia: Etreuss, 2006. p. 133-156.

TORRES, Guillermo. El desarrollo rural sustentable y el programa de desarrollo rural en México. En: VII Congreso Latinoamericano de Sociología Rural. ALASRU. Quito, Ecuador, Noviembre 2006. p. 20-24.

UC-HERNÁNDEZ, Cruz; MATUS, Jaime. Productividad y penetración Financiera Rural en México: 19782002. En: Agrociencia, 40, p. 805-812, 2006.

VERA, José; PEÑA, Martha. Desarrollo, estimulación y estrés de la crianza en infantes rurales de México. En: Apuntes de Psicología, Universidad de Sevilla y el Colegio Oficial Psicólogos, 23:3, p. 305-319, 2005. 